\title{
An integrative examination of general personality dysfunction in a large community sample
}

MICHAEL PASCAL HENGARTNER ${ }^{1}$, FILIP DE FRUYT ${ }^{2}$, STEPHANIE RODGERS ${ }^{3}$, MARIO MÜLLER ${ }^{3}$, WULF RÖSSLER ${ }^{3,4}$ AND VLADETA AJDACIC-GROSS ${ }^{3},{ }^{1}$ Department of Applied Psychology, Zurich University of Applied Sciences (ZHAW), Zurich, Switzerland; ${ }^{2}$ Department of Developmental, Personality and Social Psychology, Ghent University, Ghent, Belgium; ${ }^{3}$ Department of Psychiatry, Psychotherapy and Psychosomatics, University of Zurich, Zurich, Switzerland; ${ }^{4}$ Institute of Psychiatry, Laboratory of Neuroscience (LIM 27), University of Sao Paulo, Sao Paulo, Brazil

\section{ABSTRACT}

Recently, the severity of general personality dysfunction has gained broad interest in personality disorder (PD) research. We analysed data of 511 participants aged 20-41 years from a comprehensive psychiatric survey in the general population of Zurich, Switzerland. We added the trait-scores from all DSM-IV PDs, as assessed by a self-report questionnaire, to provide a measure of general personality dysfunction. Adjusting for the Big Five personality domains as a proxy for stylistic PD elements, this composite PD score exhibited strong associations with neuroticism and schizotypy. General personality dysfunction additionally revealed a moderate detrimental association with psychosocial functioning and a strong effect on coping resources, on heavy drinking and drug use and on most psychopathological syndromes. Of particular interest is the strong association with total psychopathological distress and co-occurrence of multiple disorders, suggesting that increasing PD severity relates to the degree of global impairment independent of specific PD traits. Discussed herein are implications for public mental health policies, classification, conceptualization and treatment of PDs. Copyright @ 2014 John Wiley $\mathcal{E}$ Sons, Ltd.

\section{Introduction}

A growing body of evidence indicates that a general personality pathology factor may load on all higherorder personality disorder (PD) traits (Hengartner, Ajdacic-Gross, Rodgers, Müller, \& Rössler, 2014; Wright et al., 2012). In support of this view, Kendler et al. (2008) found a genetic factor that pre-disposes to general PD pathology. Such a factor comprises deficits in core personality functions that are common to all PDs and substantially accounts for the high co-occurrence of $\mathrm{PD}$ diagnoses (Bornstein \& Huprich, 2011; Clark, 2007). Although there are different approaches to defining and conceptualizing the severity of general personality pathology, most PD experts agree that the assessment of a core personality dysfunction would constitute a fundamental improvement to PD nosology (Bornstein \& Huprich, 2011; Crawford, Koldobsky, Mulder, \& Tyrer, 2011; Livesley, 2011).

Basically, there exist two broad and rather independent conceptualizations of a general PD 
factor. The first resulted in the definition for DSM-5 section III, and the second is intended to be part of the PD definition in the upcoming ICD-11. For DSM-5 section III, a theoretically informed, deductive approach was chosen. Bender, Morey, and Skodol (2011) conducted a systematic review of the literature in search of assessment tools that depict general personality functioning. They inferred from their search that general personality pathology might be reduced to identity, self-direction, empathy and intimacy. They conceived those four dimensions as a means of assessing the severity of personality pathology, and divided into a clinician rating of self (identity and self-direction) and interpersonal (empathy and intimacy) functioning, this conceptualization was finally proposed as a general PD criterion for DSM-5 section III (Skodol, 2012).

Contrariwise, the conception of a general PD factor for the upcoming ICD-11 was rather atheoretical and mainly rests upon the inductive empirical work of the British psychiatrist Peter Tyrer and his colleagues (Tyrer, 2005; Tyrer \& Johnson, 1996). According to their notion, PDs should globally be classified with respect to severity. Severity was initially measured by counting PD diagnoses within and across PD clusters. Because ICD-11 aims at introducing a dimensional PD trait-model, the gateway criterion of PD severity was subsequently conceived as a one-dimensional rating of a person's functional impairment (Tyrer et al., 2011). Although the conceptualization of the two dimensions of personality functioning (DSM-5 section III) and the single dimension of global PD severity (ICD-11) is somehow different, they substantially coincide in a way that they both assess the degree of person's functional impairment.

Morey et al. (2011) found that the level of general impairment in self and interpersonal personality functioning correlates highly with the number of PD features and that it differentiates well between subjects without PD diagnosis, with only one PD diagnosis, and with several co-occurring PD diagnoses. Taking the sum of PD criteria as an index of PD severity, Hopwood et al. (2011) found that such a general factor explains considerably more variance in global functioning and measures of psychosocial functioning than five specific higher-order PD traits. Tyrer (2005) as well as Yang, Coid, and Tyrer (2010), using their approach of counting PD diagnoses within and across PD clusters, likewise reported greater psychosocial impairment with increasing PD severity. Finally, in our own work, we demonstrated substantial associations between total PD trait pathology and various interpersonal (Hengartner, Müller, Rodgers, Rössler, \& Ajdacic-Gross, 2014a) and occupational outcomes (Hengartner, Müller, Rodgers, Rössler, \& Ajdacic-Gross, 2014b). Those studies are in line with many experts' opinion that the severity of PD is clearly the better predictor of therapeutic outcomes than the type of PD (Bornstein, 1998).

The aim of the present study was to analyse the predictive value of a measure of general personality dysfunction in a large sample of the general population by focusing on a multitude of clinically relevant outcomes/covariates. We hypothesized that general personality dysfunction would considerably relate to psychosocial and psychopathological impairment independent of specific personality traits.

\section{Methods}

\section{Study design and sampling}

This study was conducted as part of the Epidemiology Survey of the Zurich Programme for Sustainable Development of Mental Health Services (ZInEP; in German: 'Zürcher Impulsprogramm zur nachhaltigen Entwicklung der Psychiatrie') (Ajdacic-Gross et al., 2014), a research and health-care programme involving several psychiatric research divisions and mental health services from the canton of Zurich, Switzerland. The Epidemiology Survey is one of the various ZInEP subprojects and consists of four components: (1) a short telephone screening; (2) a comprehensive semi-structured face-to-face interview followed by self-report questionnaires; (3) tests in the sociophysiological laboratory; and (4) a longitudinal survey. For the present study, we used only 
data from components (1) and (2). The telephone screening and semi-structured interviews started in August 2010, the tests at the sociophysiological laboratory in February 2011 and the longitudinal survey in April 2011. The screening ended in May 2012 and all other components in September 2012. Detailed information about the ZInEP Epidemiology Survey is provided by Ajdacic-Gross et al. (2014).

First, a total of 9829 Swiss men and women aged 20-41 years at the onset of the survey and considered representative of the general population of that age range of the canton of Zurich, Switzerland, were screened by computer assisted telephone interview (CATI) using the Symptom Checklist 27 (SCL-27) (Hardt, Egle, Kappis, Hessel, \& Brähler, 2004). All participants were randomly chosen through the resident registration offices of all municipalities of the canton of Zurich. Residents without Swiss nationality were excluded from the survey. The CATI was conducted by Growth for Knowledge (GfK), a major market and field research institute, in accordance with instructions from the ZInEP research team. The overall response rate was $53.6 \%$. Reasons for non-response were only telephone responder, incorrect telephone number, unavailability during the study period and refusal by a third person or the target person. In cases where potential subjects were available by telephone, the response rate was $73.9 \%$.

Second, 1500 subjects were randomly selected from the initial screening sample for subsequent face-to-face interviews. We applied a stratified sampling procedure including $60 \%$ high-scorers (scoring above the 75th percentile of the GSI of the SCL-27) and 40\% low-scorers (scoring below the 75th percentile of the GSI). The basic sampling design was adapted from the longitudinal Zurich cohort-study (Angst et al., 2005) and was chosen to enrich the sample with subjects at high-risk of mental disorders. Such a two-phase procedure with initial screening and subsequent interview with a stratified subsample is fairly common in epidemiological surveys (Dunn, Pickles, Tansella, \& Vazquez-Barquero, 1999).
Face-to-face interviews were conducted by experienced and extensively trained clinical psychologists. The interviews took place either at the participants' homes or at the University Hospital of Psychiatry in Zurich. All participants who completed the semi-structured interview were required to complete additional questionnaires. For this purpose, the sample was randomly divided into subsamples focusing either on psychosis $(N=820)$ or on PDs $(N=680)$. Out of a total of 680 subjects in the subsample focusing on PDs, $169(24.9 \%)$ refused to return or to complete all questionnaires required for the present study, resulting in a reduced final sample size of $N=511$ (284 female; 227 male; mean age: $29.6 \pm 6.7$ years).

The ZInEP Epidemiology Survey was approved by the ethics committee of the canton of Zurich (KEK) as fulfilling all legal and data privacy protection requirements and is in strict accordance with the declaration of Helsinki of the World Medical Association. All participants gave their written informed consent.

\section{Instruments and measures}

To measure dimensional PD trait-scores, we used the Assessment of DSM-IV Personality Disorders Questionnaire (ADP-IV) (Schotte \& de Doncker, 1994). For the ZInEP Epidemiology Survey, the German translation by Doering et al. (2007) was applied. The ADP-IV design allows a dimensional trait-score and a categorical PD diagnosis for each of the DSM-IV PDs. The ADP-IV is a paperpencil self-report instrument consisting of 94 items representing the 80 criteria of the 10 DSM-IV PDs and the 14 research criteria of the depressive and the passive-aggressive PDs. Each trait-question is rated on a 7-point Likert scale. Internal consistency of the ADP-IV dimensional PD scales is good (Doering et al., 2007; Schotte, de Doncker, Vankerckhoven, Vertommen, \& Cosyns, 1998) and test-retest reliability and concurrent validity of the dimensional ADP-IV trait-scores is also satisfactory (Doering et al., 2007; Schotte et al., 1998). Most importantly, the ADP-IV showed 
good concordance with the SCID-II interview (Schotte et al., 2004) and may therefore be considered an economical and valid alternative to semi-structured interviews.

In keeping with Hopwood et al. (2011), we provided a general personality dysfunction score by adding all DSM-IV trait-scores. This approach is consistent with the notion that the sum-score of all PD criteria captures the very nature of global and unspecific personality impairment, that is, the variance shared among PD dimensions. However, such a composite score is still composed of specific PD traits; therefore, it needs to be adjusted for those stylistic elements to provide an independent and unbiased measure. In this respect, our conceptualization of a one-dimensional measure of general personality pathology more closely resembles the approach proposed for inclusion in ICD-11 than the rating of self and interpersonal functioning delineated by the DSM-5 work group. To use consistent terminology hereafter, we label that core dimension of PD pathology 'general personality dysfunction', and in the following, we will use this term throughout.

We briefly introduce all other instruments applied in the present study, which comprise the almost complete set of questionnaires applied in the ZInEP Epidemiology Survey. This approach was chosen to cover the broadest possible range of psychosocial covariates provided by our data. All instruments showed good validity and reliability. For more information, see the respective literature indicated in the succeeding text.

The SCL-27 (Hardt et al., 2004) is a German short form of the well-known SCL-90-R (Derogatis, 1977) that covers a wide variety of psychopathological symptoms from the most recent 4-week period. Subjects responded according to a 5-point Likert scale. The SCL-27 contains the six subscales depressive, dysthymic, vegetative, agoraphobic, sociophobic symptoms and symptoms of mistrust. A total distress score similar to the global severity index (GSI) of the SCL-90-R is also available.

The Positive and Negative Affect Schedule (Watson, Clark, \& Tellegen, 1988) is a short self-report questionnaire that measures broad positive (10 items) and negative (10 items) affects according to a 5-point Likert scale.

The attention deficit hyperactivity disorder self-report questionnaire (ADHD-SR) (Rosler et al., 2004) was developed to assess current ADHD symptoms in adulthood. The ADHD-SR comprises 22 items rated on a 4-point Likert scale. The items are divided into four subscales, which together yield a total score.

The Big Five Inventory short form (BFI-S) (Schupp \& Gerlitz, 2008) is a German adaptation of the popular Big Five Inventory by John, Donahue, and Kentle (1991). The questionnaire consists of 15 items divided into the five broad domains neuroticism, extraversion, openness, agreeableness and conscientiousness. The items are rated on a 7-point Likert scale.

The Sensation Seeking Scale form V (SSS-V) (Zuckerman, 1994) defines a broad personality trait marked by striving for novel and complex experiences and the willingness to take physical, social, legal and financial risks for the sake of such experiences. The SSS-V consists of four subscales, that is, thrill and adventure seeking, disinhibition, experience seeking and boredom susceptibility. For this study, a German translation of the SSS-V (Beauducel, Strobel, \& Brocke, 2003) was applied.

The brief form of the Schizotypal Personality Questionnaire (SPQ-B) (Raine \& Benishay, 1995) consists of 22 items and measures three factors of schizotypy, namely cognitive-perceptual (e.g. paranoid ideation, illusionary perception), interpersonal (e.g. lack of close friends, social withdrawal) and disorganized (e.g. eccentric behaviour, odd mannerisms). For this study, a German translation was used (Klein, Andresen, \& Jahn, 1997).

Different coping styles were assessed with the brief Coping Orientation to Problems Experienced (Brief COPE) (Carver, 1997). The Brief COPE is a self-report questionnaire comprising 28 items, which are rated on a 4-point Likert scale. The Brief COPE allows measuring features of emotion-focused coping (e.g. to search for social support and accept reality), problem-focused coping (e.g. to conceive 
problem solving strategies) and dysfunctional coping (e.g. to distract oneself with alcohol use, denial) (Cooper, Katona, \& Livingston, 2008).

The Trier Inventory for the Assessment of Chronic Stress (Schulz \& Schlotz, 1999) was developed to assess chronic stress in various domains of daily life such as work overload, worries, lack of social recognition or work discontent. From this questionnaire, a short form called Screening Scale for Chronic Stress (SSCS) (Schulz, Schlotz, \& Becker, 2004) was derived. The SSCS consists of 12 items rated on a 5 -point Likert scale and provides a global measure of chronic stress.

The Connor-Davidson Resilience Scale (CDRISC) (Connor \& Davidson, 2003) captures a global measure of resilience, a construct defined as the ability to cope with stress and resistance against adverse experiences (Richardson, 2002). The CD-RISC consists of 25 items rated on a 5-point Likert scale.

Education level (high vs. low) was assessed during the CATI by asking the participants to indicate their highest educational degree. These qualifications were subsequently categorized as low (if the highest attainment corresponded to a high school diploma) and high (if it corresponded to a qualification above high school). All other socio-demographics, substance use variables and psychiatric diagnoses were assessed during the semi-structured interview with the 'Structured Psychopathological Interview and Rating of the Social Consequences of Psychological Disturbances for Epidemiology' (SPIKE) (Angst, Dobler-Mikola, \& Binder, 1984). This instrument was developed for epidemiological surveys in psychiatric research and evaluates data about socio-demography, somatic syndromes, psychopathology, substance use, medication, health services, impairment and social activity. Its reliability and validity have been reported elsewhere (Angst et al., 2005). Twelve-month prevalence diagnoses of mental disorders according to DSM-IV criteria comprised major depression episode, agoraphobia, simple phobia, social phobia, obsessive-compulsive disorder, panic disorder and alcohol use disorder (abuse or dependence). In addition, a measure of co-occurrence was computed by adding the number of co-occurring disorders.

\section{Statistical analysis}

First, because values on all continuous PD traitscores as well as Big Five traits were missing completely at random (MCAR) according to Little's MCAR test $\left(\chi^{2}=189.972\right.$, d.f. $=182$, $p=0.328)$, we conducted a missing value analysis (MVA). No variable was missing in more than maximally nine subjects (1.8\%). MVA was carried out with the full information maximum likelihood estimation, an MVA procedure recommended by Schafer and Graham (2002). Afterwards, we conducted a series of different regression models. The general personality dysfunction score was always included as the independent variable. In order to adjust each analysis for sex and age, these variables were also included as covariates. Because the main objective of this study was to separate general personality dysfunction from the effects of PD traits, we additionally adjusted all models for the Big Five domains. The latter closely correspond to the following higher-order PD domains: negative affectivity, detachment, antagonism, disinhibition and psychoticism (Thomas et al., 2013). By this means, we were able to demonstrate the associations of a general personality dysfunction factor above and beyond the variance explained by stylistic elements. Depending on the respective outcomes of the analyses, we fitted binary logistic regression models for dichotomous outcomes, multinomial logistic regression models for outcomes with more than two categories and robust generalized linear regression models for continuous outcomes. Results of the binary and logistic regression analyses were reported with odds ratios (OR) and their corresponding 95\% confidence intervals $(\mathrm{CI})$, whereas the generalized linear regression analyses applied standardized regression coefficients $(\beta)$ and their corresponding 95\% CI. All analyses were performed with SPSS 20 (Armonk, NY: IBM Corp.) for Macintosh. 


\section{Results}

The general personality dysfunction raw-score ranged from 10.00 to 54.24 and was approximately normally distributed with a mean of 22.41 and a standard deviation of 6.96. For all subsequent analyses, this score was standardized using the $z$-transformation. We also computed an alternative measure of general PD dysfunction by using the first unrotated component of a principal component analysis of all 10 PD trait-scores. The correlation between these two alternative measures of general personality dysfunction was $r=0.99$. The bivariate correlation between general personality dysfunction and the DSM-IV PD traitscores was substantial (mean $r=0.76$ ). The correlation between general personality dysfunction and the specific PD traits was $r=0.81$ for paranoid PD, $r=0.62$ for schizoid PD, $r=0.84$ for schizotypal PD, $r=0.64$ for antisocial PD, $r=0.85$ for borderline PD, $r=0.76$ for histrionic PD, $r=0.77$ for narcissistic PD, $r=0.80$ for avoidant PD, $r=0.79$ for dependent PD and $r=0.76$ for obsessivecompulsive PD. General personality dysfunction was not related to sex (female: -0.046 ; male: 0.058; $p=0.239$ ) but was weakly correlated with age $(r=-0.094 ; p=0.033)$. Because of significant interrelations of these variables with various outcomes included in the study, in addition to the Big Five traits, all subsequent analyses were also adjusted for sex and age.

Associations with socio-demographics and substance use are indicated in Table 1. General personality dysfunction was related to being unmarried, living alone, having no children and no partner, having a low education level and being unemployed. All these associations were modest and within the range of small-to-medium effect sizes. The strongest effect was found for partnership, where a 1-standard deviation increase in general personality dysfunction decreased the odds of having a partner by $75 \%$. The standardized mean difference between having a partner and not having a partner was 0.38, which represents a small-tomedium effect size according to Cohen's $d$.
General personality dysfunction substantially predicted heavy drinking (more than four standard drinks per occasion) and cannabis as well as other drug use. The effect size was particularly strong for quantity of alcohol use, where a 1-standard deviation increase in general personality dysfunction increased the odds of drinking more than four standard units by $150 \%$. Expressed in standardized mean differences, the disparity in general personality dysfunction between drinking 0-2 units and drinking more than four units was 0.64 (mediumto-large effect size). Cohen's $d$ for the mean difference between no drug use and other drug use was 0.61 .

The associations between general personality dysfunction and psychopathological syndromes are reported in Table 2. All associations with the SCL-27 subscales, both PANAS subscales and the ADHD total score, were substantial, with $\beta=0.300$ indicating a medium effect size and $\beta=0.500$ representing a large effect size. The strong association between SCL-27 global severity and general personality dysfunction $(\beta=0.491)$ in addition indicates that increased general personality dysfunction predicts global psychopathological symptom load across various syndromes.

The considerable associations between general personality dysfunction and psychopathological syndromes were complemented with associations with psychiatric diagnoses (Table 3). A 1-standard deviation increase in general personality dysfunction in particular increased the odds of having 12-month prevalence diagnosis of major depression $(\mathrm{OR}=1.95)$ and alcohol use disorder $(\mathrm{OR}=2.16)$. Social phobia was the only anxiety disorder that showed a significant association with general personality dysfunction. A considerably large effect size was found with respect to co-occurrence, where the number of concurrent diagnoses steadily increased the level of general personality dysfunction. Whereas the standardized mean difference between no disorder and one disorder was 0.28 , it was 0.38 between no disorder and two disorders and even 0.84 between no disorder and more than 
Table 1: Associations of general personality dysfunction with socio-demographics and substance use, adjusted for sex, age and Big Five personality traits

\begin{tabular}{|c|c|c|c|c|}
\hline & & OR & $95 \% \mathrm{CI}$ & Significance \\
\hline \multirow[t]{2}{*}{ Marital status } & Married $(N=134)$ & 0.70 & $0.51 ; 0.95$ & 0.022 \\
\hline & Unmarried $(N=371)$ & Ref. & & \\
\hline \multirow[t]{2}{*}{ Housing } & Community $(N=404)$ & 0.70 & $0.51 ; 0.88$ & 0.004 \\
\hline & Alone $(N=99)$ & Ref. & & \\
\hline \multirow[t]{2}{*}{ Children } & Yes $(N=121)$ & 0.66 & $0.47 ; 0.93$ & 0.017 \\
\hline & No $(N=389)$ & Ref. & & \\
\hline \multirow[t]{2}{*}{ Partner } & Yes $(N=340)$ & 0.57 & $0.45 ; 0.73$ & 0.000 \\
\hline & No $(N=171)$ & Ref. & & \\
\hline \multirow[t]{2}{*}{ Education } & $\operatorname{High}(N=208)$ & 0.78 & $0.61 ; 0.99$ & 0.041 \\
\hline & Low $(N=302)$ & Ref. & & \\
\hline \multirow[t]{2}{*}{ Unemployment } & Yes $(N=49)$ & 1.46 & $1.03 ; 2.06$ & 0.034 \\
\hline & No $(N=462)$ & Ref. & & \\
\hline \multirow[t]{3}{*}{ Smoking } & Daily $(N=109)$ & 1.29 & $1.00 ; 1.67$ & 0.053 \\
\hline & Occasional $(N=33)$ & 1.12 & $0.72 ; 1.73$ & 0.619 \\
\hline & Non $(N=369)$ & Ref. & & \\
\hline \multirow[t]{3}{*}{ Alcohol frequency } & $>3$ per week $(N=51)$ & 0.96 & $0.66 ; 1.40$ & 0.834 \\
\hline & $1-3$ per week $(N=264)$ & 0.78 & $0.62 ; 0.98$ & 0.037 \\
\hline & $<1$ per week $(N=195)$ & Ref. & & \\
\hline \multirow[t]{3}{*}{ Alcohol quantity } & $>4$ st. drink $(N=52)$ & 2.50 & $1.71 ; 3.66$ & 0.000 \\
\hline & $3-4$ st. drink $(N=120)$ & 1.26 & $0.94 ; 1.66$ & 0.109 \\
\hline & $0-2$ st. drink $(N=339)$ & Ref. & & \\
\hline \multirow[t]{3}{*}{ Drug use } & Other drugs $(N=29)$ & 2.12 & $1.37 ; 3.26$ & 0.001 \\
\hline & Only cannabis $(N=81)$ & 1.62 & $1.20 ; 2.19$ & 0.002 \\
\hline & No drugs $(N=401)$ & Ref. & & \\
\hline
\end{tabular}

OR, odds ratio; $\mathrm{CI}$, confidence interval.

Table 2: Associations of general personality dysfunction with psychopathological syndromes, adjusted for sex, age and Big Five personality traits

\begin{tabular}{lllll}
\hline & & $\beta$ & $95 \%$ CI & Significance \\
\hline SCL-27 & Depressive symptoms $(N=511)$ & 0.372 & $0.274 ; 0.469$ & 0.000 \\
& Dysthymic symptoms $(N=510)$ & 0.345 & $0.248 ; 0.442$ & 0.000 \\
& Vegetative symptoms $(N=511)$ & 0.290 & $0.191 ; 0.389$ & 0.000 \\
& Agoraphobic symptoms $(N=511)$ & 0.308 & $0.181 ; 0.434$ & 0.000 \\
& Sociophobic symptoms $(N=510)$ & 0.424 & $0.329 ; 0.519$ & 0.000 \\
& Mistrust symptoms $(N=509)$ & 0.467 & $0.379 ; 0.554$ & 0.000 \\
PANAS & Global severity $(N=508)$ & 0.491 & $0.406 ; 0.576$ & 0.000 \\
& Positive affect $(N=498)$ & -0.232 & $-0.349 ;-0.115$ & 0.000 \\
ADHD-SR & Negative affect $(N=501)$ & 0.401 & $0.311 ; 0.491$ & 0.000 \\
& ADHD symptoms $(N=491)$ & 0.525 & $0.418 ; 0.632$ & 0.000 \\
\hline
\end{tabular}

SCL-27, Symptom Checklist 27; PANAS, Positive and Negative Affect Schedule; ADHD-SR, attention deficit hyperactivity disorder self-report questionnaire; CI, confidence interval. 
Table 3: Associations of general personality dysfunction with psychiatric diagnoses, adjusted for sex, age and Big Five personality traits

\begin{tabular}{|c|c|c|c|c|}
\hline & & OR & $95 \% \mathrm{CI}$ & Significance \\
\hline SPIKE & $\begin{array}{l}\text { Major depression }(N=136) \\
\text { No major depression }(N=375) \\
\text { Agoraphobia }(N=25) \\
\text { No agoraphobia }(N=486) \\
\text { Simple phobia }(N=84) \\
\text { No simple phobia }(N=427) \\
\text { Social phobia }(N=48) \\
\text { No social phobia }(N=463) \\
\text { OCD }(N=42) \\
\text { No OCD }(N=469) \\
\text { Panic disorder }(N=23) \\
\text { No panic disorder }(N=488) \\
\text { Alcohol use disorder }(N=52) \\
\text { No alcohol use disorder }(N=459) \\
>2 \text { disorders }(N=40) \\
2 \text { disorders }(N=68) \\
1 \text { disorder }(N=142) \\
\text { No disorder }(N=261)\end{array}$ & $\begin{array}{l}1.95 \\
\text { Ref. } \\
1.33 \\
\text { Ref. } \\
1.17 \\
\text { Ref. } \\
1.42 \\
\text { Ref. } \\
0.98 \\
\text { Ref. } \\
1.29 \\
\text { Ref. } \\
2.16 \\
\text { Ref. } \\
2.60 \\
1.85 \\
1.67 \\
\text { Ref. }\end{array}$ & $\begin{array}{l}1.49 ; 2.56 \\
0.86 ; 2.07 \\
0.88 ; 1.54 \\
1.01 ; 2.00 \\
0.69 ; 1.41 \\
0.82 ; 2.02 \\
1.49 ; 3.13 \\
1.68 ; 4.03 \\
1.31 ; 2.62 \\
1.25 ; 2.20\end{array}$ & $\begin{array}{l}0.000 \\
0.203 \\
0.284 \\
0.045 \\
0.929 \\
0.279 \\
0.000 \\
0.000 \\
0.001 \\
0.000\end{array}$ \\
\hline
\end{tabular}

OR, odds ratio; CI, confidence interval; SPIKE, Structured Psychopathological Interview and Rating of the Social Consequences of Psychological Disturbances for Epidemiology, OCD, obsessive-compulsive disorder.

two disorders. The latter corresponds to a large effect size.

As a next step, we regressed coping resources and various personality traits on general personality dysfunction (Table 4). General personality dysfunction was strongly associated with dysfunctional coping $(\beta=0.505)$ and moderately with chronic stress $(\beta=0.445)$ as measured by the Brief COPE and SSCS respectively. The association with resilience according to the CD-RISC total score was negative and rather modest $(\beta=-0.273$; small-to-medium effect size).

Among the BFI-S traits, general personality dysfunction was mainly related to neuroticism ( $\beta=0.403$; medium-to-large effect size), followed by extraversion, agreeableness and openness $(\beta=-0.323,-0.245$ and 0.235 respectively). With respect to the SSS-V, only weak associations with boredom susceptibility $(\beta=0.153)$ and disinhibition $(\beta=0.115)$ emerged, whereas the associations with all SPQ-B subscales represented large effects $(0.526<\beta>0.576)$.

\section{Discussion}

In this study, we examined the association between general personality dysfunction and various measures of psychosocial functioning and mental disorders in a large general population-based sample. We included a multitude of different measures from a broad range of clinically relevant domains encompassing socio-demographics, substance use, psychopathology, personality and coping. A general personality dysfunction score was obtained through adding the trait-scores of all 10 DSM-IV PDs.

Most importantly, by adjusting all associations for the Big Five personality traits (which depict stylistic elements), we provide compelling evidence that a general personality dysfunction factor is independent of specific traits. That is, this general factor substantially explains variance above and beyond specific PD traits. This is an important finding with implications for clinical practice and mental health policies. For instance, until recently 
Table 4: Associations of general personality dysfunction with coping resources and personality traits, adjusted for sex, age and Big Five personality traits

\begin{tabular}{llccc}
\hline & & $\beta$ & $95 \% \mathrm{CI}$ & Significance \\
\hline Brief COPE & Problem-focused coping $(N=498)$ & -0.049 & $-0.156 ; 0.057$ & 0.367 \\
& Emotion-focused coping $(N=501)$ & -0.055 & $-0.172 ; 0.061$ & 0.350 \\
& Dysfunctional coping $(N=503)$ & 0.505 & $0.408 ; 0.602$ & 0.000 \\
SSCS & Chronic stress $(N=497)$ & 0.445 & $0.354 ; 0.535$ & 0.000 \\
CD-RISC & Resilience $(N=505)$ & -0.273 & $-0.376 ;-0.169$ & 0.000 \\
BFI-S $*$ & Neuroticism $(N=511)$ & 0.403 & $0.325 ; 0.482$ & 0.000 \\
& Extraversion $(N=511)$ & -0.323 & $-0.418 ;-0.227$ & 0.000 \\
& Openness $(N=511)$ & 0.235 & $0.145 ; 0.325$ & 0.000 \\
& Conscientiousness $(N=511)$ & 0.018 & $-0.097 ; 0.132$ & 0.762 \\
SSS-V & Agreeableness $(N=511)$ & -0.245 & $-0.356 ;-0.135$ & 0.000 \\
& Thrill and adventure seeking $(N=496)$ & 0.035 & $-0.062 ; 0.133$ & 0.479 \\
& Disinhibition $(N=484)$ & 0.115 & $0.013 ; 0.217$ & 0.027 \\
& Experience seeking $(N=492)$ & -0.059 & $-0.153 ; 0.035$ & 0.216 \\
SPQ-B & Boredom susceptibility $(N=489)$ & 0.153 & $0.054 ; 0.253$ & 0.003 \\
& Cognitive-perceptual $(N=500)$ & 0.575 & $0.490 ; 0.660$ & 0.000 \\
& Interpersonal $(N=495)$ & 0.529 & $0.453 ; 0.605$ & 0.000 \\
& Disorganized $(N=503)$ & 0.527 & $0.449 ; 0.605$ & 0.000 \\
\hline
\end{tabular}

*The BFI trait that served as the dependent variable was not included as a covariate.

Brief COPE, brief Coping Orientation to Problems Experienced; SSCS, Screening Scale for Chronic Stress; CD-RISC, Connor-Davidson Resilience Scale; BFI-S, Big Five Inventory short form; SSS-V, Sensation Seeking Scale form V; SPQ-B, brief form of the Schizotypal Personality Questionnaire.

and on the basis of DSM-IV and ICD-10 definitions, it was mostly unknown whether someone with borderline PD traits showed marked functional impairment because of the symptomatology of the disorder or because of the severity of the disorder. With the present study, we could demonstrate that a substantial proportion of variance in psychosocial and psychopathological impairment is independent of specific PD traits and clearly attributable to general personality dysfunction, that is, to the severity of personality pathology. In this respect, Hopwood et al. (2011) argued that severity of general personality dysfunction would quantify the degree of functional impairment and PD styles would specify the manner in which dysfunction would be expressed. By separating severity from traits, PD researchers are finally able to examine issues that are crucial to the understanding of PDs, such as their high co-occurrence with substance use or mood disorders, from a new perspective. Future studies should thus consistently try to disentangle the effects of PD severity and PD traits. Finally, it has also been posited that a PD diagnosis that incorporates severity of general dysfunction and PD traits improves validity and reliability of PD assessment (Bornstein \& Huprich, 2011; Livesley, 2011). In support of this hypothesis, it has been shown that a combination of general personality dysfunction and PD traits, as incorporated in the alternative PD model in DSM-5 section III, provides incremental information about the occurrence and severity of PDs (Berghuis, Kamphuis, \& Verheul, 2013; Hopwood, Thomas, Markon, Wright, \& Krueger, 2012).

Socio-demographics were related weakly to general personality dysfunction. The strongest association was found with having no partner. Examination of substance use revealed considerable associations with cannabis use, other drug use and in particular quantity of alcohol use. The 
association between general personality dysfunction and heavy drinking corresponded to a medium-tolarge effect size. Taken together, those findings indicate that general personality dysfunction is consistently and meaningfully related to psychosocial impairment, which is in line with the literature (Hopwood et al., 2011; Yang et al., 2010).

No study has so far addressed the associations between general personality dysfunction and a broad range of psychopathological syndromes independent of stylistic PD elements. In this respect, we found a moderate association of general personality dysfunction with depressive, dysthymic, vegetative, agoraphobic, sociophobic and mistrust symptoms. Especially the associations with the latter two syndromes, which represent disorders of interpersonal functioning, nearly corresponded to large effect sizes. Those associations are particularly impressive when taking into account that all models were adjusted for neuroticism, which is an important predictor of psychopathology (Lahey, 2009). Interestingly, Morey et al. (2011) also argued that increasing severity of personality pathology might reflect interpersonal functioning deficits rather than self or identity issues. This could also be the reason why social phobia as a disorder of interpersonal functioning was the only anxiety disorder that showed a significant association with general personality dysfunction when adjusting for neuroticism.

Taken together, our findings confirm the strong link between general personality dysfunction and psychopathological symptoms reported by Yang et al. (2010) and adds to the literature on cooccurrence between disorders and continuity of PDs and other mental disorders (Krueger, 2005; Widiger, 2003). The considerably strong association between general personality dysfunction and co-occurrence with multiple DSM-IV diagnoses, representing a large effect size, merits special emphasis. This finding confirms a marked relationship between total impairment and PD severity, which has also been found with respect to the number of co-occurring PD diagnoses (Morey et al., 2011) or psychosocial functioning (Hengartner, Müller et al., 2014a, 2014b). Moreover, general personality dysfunction demonstrated a strong positive association with dysfunctional coping, a moderate relationship to chronic stress and a modest negative association with resilience. Similar findings have been reported previously (Bijttebier \& Vertommen, 1999; Hengartner, Müller, Rodgers, Rössler, \& Ajdacic-Gross, 2013; van Wijk-Herbrink, Andrea, $\&$ Verheul, 2011) and underline the high personal distress and impairment related to PDs.

Our finding that general personality dysfunction particularly relates to neuroticism confirms the results reported by Hopwood et al. (2011) and is consistent with the literature on the joint structure of normal and pathological personality (Samuel \& Widiger, 2008). There is compelling evidence that neuroticism is a core feature of general personality dysfunction and the most important personality trait underlying PD symptomatology (Hengartner, Ajdacic-Gross et al., 2014; Samuel \& Widiger, 2008). It is noteworthy that general personality dysfunction was also meaningfully related to openness. Because there is an ongoing and hot debate whether adaptive openness is related to PD traits, this finding is of particular interest to the field (see, for instance, Chmielewski, Bagby, Markon, Ring, \& Ryder, 2014 ; Widiger \& Mullins-Sweatt, 2009).

In this respect, we additionally stress the strong relationship between general personality dysfunction and all three subscales of the schizotypal personality questionnaire, that is, cognitiveperceptual aberrations, interpersonal deficits and disorganized behaviour. These findings align with results reported by Caspi et al. (2013) who found that a schizotypal factor (which they labelled 'thought disorder') is strongly correlated with a general psychopathology factor, even markedly stronger than an externalizing or internalizing factor. In contrast, the association between general personality dysfunction and sensation seeking was modest and only meaningful with respect to disinhibition and boredom susceptibility. This is attributable to the disparate associations of sensation seeking with different PD traits. Further 
analyses of the data revealed that sensation seeking was positively related to antisocial PD, but mostly unrelated to other PDs and even negatively related to dependent PD (Hengartner, Rodgers, Müller, Rössler \& Ajdacic-Gross, 2014).

In conclusion, the findings of the present study have serious implications for psychiatric nosology and epidemiology as well as clinical practice, because the severity of general personality dysfunction, first, predicts psychosocial outcomes much better than specific PD types, second, is less stigmatizing, third, more relevant for policy makers, and fourth, more easily applicable and more serviceable for researchers and practitioners (Hopwood et al., 2011; Tyrer, 2005; Yang et al., 2010). Most importantly, because general personality dysfunction and its adaptive capacity are susceptible to change in the mid-term and long term (Verheul et al., 2008), it constitutes a major target for clinical intervention (Verheul \& Herbrink, 2007), which may be particularly helpful in high-risk populations such as victims of child maltreatment (Hengartner et al., 2013).

Although we emphasize the significance of general personality dysfunction, we do advocate the assessment of a comprehensive set of individual PD traits, and vice versa, because both specific PD traits and general personality dysfunction provide incremental validity above and beyond each other (Bastiaansen, De Fruyt, Rossi, Schotte, \& Hofmans, 2013; Hopwood et al., 2012). Therefore, we suggest that the alternative PD model from section III of DSM-5 would provide greater reliability and validity than the outdated definition of DSM-IV. For now, our hope lies in the upcoming ICD-11 that it might finally introduce a dimensional PD model that separates severity from traits. A further question that is open to debate is the structure of general personality dysfunction and whether it is best conceptualized as one or several dimensions (Bastiaansen et al., 2013; Morey et al., 2011). It is also important to state that different dimensions of general personality dysfunction (for instance, self-control vs. identity integration) may show disparate associations in some PD traits (Bastiaansen et al., 2013).
Finally, the results of the present study need to be interpreted in the light of the following major limitations. First, and most importantly, because of the cross-sectional design, we cannot draw clear causal conclusions. Second, all measures relied on self-report, and results may therefore be influenced by false-positive or false-negative reports and may be biased through method effects such as social desirability. Third, the ADP-IV is a self-rating scale and therefore regarded as a screening instrument by some experts. The continuous ADP-IV trait-scores do not provide a clear threshold for clinical states, i.e. for PD diagnoses. However, because PDs are treated as continuous traitdimensions, such a distinction would be arbitrary anyway. Fourth, multiple testing increases the rate of potential $\alpha$-errors. However, because adjustment for multiple testing in turn increases the rate of $\beta$-errors markedly and in consideration of the substantial effect sizes (which suggest that those associations are not merely due to chance), we did not use an $\alpha$-correction.

\section{Acknowledgement}

The ZInEP was supported by a private foundation. The foundation had no further role in the experimental design, the collection, analysis, and interpretation of data, the writing of this report or the decision to submit this paper for publication.

\section{Conflict of interest}

There is no conflict of interest.

\section{References}

Ajdacic-Gross, V., Müller, M., Rodgers, S., Warnke, I., Hengartner, M. P., Landolt, K., Hagenmuller, F., Meier, M., Tse, L.-T., Aleksandrowitz, A., Passardi, M., Knöpfli, D., Schönfelder, H., Eisele, J., Rüsch, N., Haker, H., Kawohl, W., \& Rössler, W. (2014). The ZInEP Epidemiology Survey: background, design and methods. International Journal of Methods in Psychiatric Research, in press.

Angst, J., Dobler-Mikola, A., \& Binder, J. (1984). The Zurich study-a prospective epidemiological study of depressive, neurotic and psychosomatic syndromes. I. Problem, 
methodology. European Archives of Psychiatry and Neurological Sciences, 234(1), 13-20.

Angst, J., Gamma, A., Neuenschwander, M., Ajdacic-Gross, V., Eich, D., Rössler, W., \& Merikangas, K. R. (2005). Prevalence of mental disorders in the Zurich cohort study: a twenty year prospective study. Epidemiologia e Psichiatria Sociale, 14(2), 68-76.

Bastiaansen, L., De Fruyt, F., Rossi, G., Schotte, C., \& Hofmans, J. (2013). Personality disorder dysfunction versus traits: structural and conceptual issues. Personal Disord, 4(4), 293-303. doi: 10.1037/per0000018

Beauducel, A., Strobel, A., \& Brocke, B. (2003). Psychometrische Eigenschaften und Normen einer deutschsprachigen Fassung der Sensation SeekingSkalen, Form V. Diagnostica, 49(2), 61-72.

Bender, D. S., Morey, L. C., \& Skodol, A. E. (2011). Toward a model for assessing level of personality functioning in DSM-5, part I: a review of theory and methods. Journal of Personality Assessment, 93(4), 332-346. doi: 10.1080/ 00223891.2011 .583808

Berghuis, H., Kamphuis, J. H., \& Verheul, R. (2013). Specific personality traits and general personality dysfunction as predictors of the presence and severity of personality disorders in a clinical sample. Journal of Personality Assessment. doi: 10.1080/00223891.2013.834825

Bijttebier, P., \& Vertommen, H. (1999). Coping strategies in relation to personality disorders. Personality and Individual Differences, 26(5), 847-856.

Bornstein, R. F. (1998). Reconceptualizing personality disorder diagnosis in the DSM-V: the discriminant validity challenge. Clinical Psychology Science Practice, 5(3), 333-343.

Bornstein, R. F., \& Huprich, S. K. (2011). Beyond dysfunction and threshold-based classification: a multidimensional model of personality disorder diagnosis. Joumal of Personality Disorders, 25(3), 331-337. doi: 10.1521/pedi.2011.25.3.331

Carver, C. S. (1997). You want to measure coping but your protocol's too long: consider the brief COPE. International Journal of Behavioral Medicine, 4(1), 92-100.

Caspi, A., Houts, R. M., Belsky, D. W., Goldman-Mellor, S. J., Harrington, H., Israel, S., Meier, M. H., Ramrakha, S., Shalev, I., Poulton, R., \& Moffitt, T. E., (2013). The $\mathrm{p}$ factor: one general psychopathology factor in the structure of psychiatric disorders? Clinical Psychological Science. doi: 10.1177/2167702613497473

Chmielewski, M., Bagby, R. M., Markon, K., Ring, A. J., \& Ryder, A. G. (2014). Openness to experience, intellect, schizotypal personality disorder, and psychoticism: resolving the controversy. Journal of Personality Disorders. doi: 10.1521/pedi_2014_28_128

Clark, L. A. (2007). Assessment and diagnosis of personality disorder: perennial issues and an emerging reconceptualization. Annual Review of Psychology, 58, 227-257. doi: 10.1146/annurev.psych.57.102904.190200
Connor, K. M., \& Davidson, J. R. (2003). Development of a new resilience scale: the Connor-Davidson Resilience Scale (CD-RISC). Depression and Anxiety, 18(2), 76-82. doi: $10.1002 /$ da. 10113

Cooper, C., Katona, C., \& Livingston, G. (2008). Validity and reliability of the brief COPE in carers of people with dementia: the LASER-AD Study. Journal of Nervous and Mental Disease, 196(11), 838-843. doi: 10.1097/NMD.0b013e31818b504c

Crawford, M. J., Koldobsky, N., Mulder, R., \& Tyrer, P. (2011). Classifying personality disorder according to severity. Journal of Personality Disorders, 25(3), 321-330. doi: 10.1521/pedi.2011.25.3.321

Derogatis, R. L. (1977). Symptom Checklist 90, R-version manual I: scoring, administration, and procedures for the SCL-90. Baltimore, MD: Johns Hopkins Press.

Doering, S., Renn, D., Hofer, S., Rumpold, G., Smrekar, U., Janecke, N., Schatz, D. S., Schotte, C. K. W., De Doncker, D. A., \& Schussler, G. (2007). Validation of the German version of the assessment of DSM-IV Personality Disorders (ADP-IV) Questionnaire. Zeitschrift für Psychosomatische Medizin und Psychotherapie, 53(2), 111-128.

Dunn, G., Pickles, A., Tansella, M., \& Vazquez-Barquero, J. L. (1999). Two-phase epidemiological surveys in psychiatric research. British Journal of Psychiatry, 174, 95-100.

Hardt, J., Egle, U. T., Kappis, B., Hessel, A., \& Brähler, E. (2004). Symptom Checklist SCL-27: results of a representative German survey. Psychotherapie, Psychosomatik, Medizinische Psychologie, 54(5), 214-223. doi: 10.1055/s2003-814786

Hengartner, M. P., Ajdacic-Gross, V., Rodgers, S., Müller, M., \& Rössler, W. (2014). The joint structure of normal and pathological personality: further evidence for a dimensional model. Comprehensive Psychiatry, 55(3), 667-674. doi: 10.1016/j.comppsych.2013.10.011

Hengartner, M. P., Müller, M., Rodgers, S., Rössler, W., \& Ajdacic-Gross, V. (2013). Can protective factors moderate the detrimental effects of child maltreatment on personality functioning? Journal of Psychiatric Research, 47(9), 1180-1186. doi: 10.1016/j.jpsychires.2013.05.005

Hengartner, M. P., Müller, M., Rodgers, S., Rössler, W., \& Ajdacic-Gross, V. (2014a). Interpersonal functioning deficits in association with DSM-IV personality disorder dimensions. Social Psychiatry and Psychiatric Epidemiology, 49(2), 317-325. doi: 10.1007/s00127-013-0707-x

Hengartner, M. P., Müller, M., Rodgers, S., Rössler, W., \& Ajdacic-Gross, V. (2014b). Occupational functioning and work impairment in association with personality disorder trait-scores. Social Psychiatry and Psychiatric Epidemiology, 49(2), 327-335. doi: 10.1007/s00127013-0739-2

Hengartner, M. P., Rodgers, S., Müller, M., Rössler, W., \& Ajdacic-Gross, V. (2014). Substance use in association 
with personality disorder traits and the effects mediated by dysfunctional coping and sensation seeking. Annals of Psychiatry and Mental Health, 2(1), 1005.

Hopwood, C. J., Malone, J. C., Ansell, E. B., Sanislow, C. A., Grilo, C. M., McGlashan, T. H., Pinto, A., Markowitz, J. C., Shea, M. T., Skodol, A. E., Gunderson, J. G., Zanarini, M. C., \& Morey, L. C. (2011). Personality assessment in DSM-5: empirical support for rating severity, style, and traits. Journal of Personality Disorders, 25(3), 305-320. doi: 10.1521/pedi.2011.25.3.305

Hopwood, C. J., Thomas, K. M., Markon, K. E., Wright, A. G., \& Krueger, R. F. (2012). DSM-5 personality traits and DSM-IV personality disorders. Journal of Abnormal Psychology, 121(2), 424-432. doi: 10.1037/a0026656

John, O. P., Donahue, E. M., \& Kentle, R. L. (1991). The Big Five Inventory - Versions $4 a$ and 54. Berkeley, CA: University of California, Berkeley, Institute of Personality and Social Research.

Kendler, K. S., Aggen, S. H., Czajkowski, N., Roysamb, E., Tambs, K., Torgersen, S., Neale, M. C., \& ReichbornKjennerud, T. (2008). The structure of genetic and environmental risk factors for DSM-IV personality disorders: a multivariate twin study. Archives of General Psychiatry, 65 (12), 1438-1446. doi: 10.1001/archpsyc.65.12.1438

Klein, C., Andresen, B., \& Jahn, T. (1997). Erfassung der schizotypen Persönlichkeit nach DSM-III-R: Psychometrische Eigenschaften einer autorisierten deutschsprachigen Übersetzung des Schizotypal Personality Questionnaire (SPQ) von Raine. Diagnostica, 43, 347-369.

Krueger, R. F. (2005). Continuity of axes I and II: toward a unified model of personality, personality disorders, and clinical disorders. Journal of Personality Disorders, 19(3), 233-261.

Lahey, B. B. (2009). Public health significance of neuroticism. American Psychologist, 64(4), 241-256. doi: 10.1037/ a0015309

Livesley, W. J. (2011). An empirically-based classification of personality disorder. Journal of Personality Disorders, 25(3), 397-420. doi: 10.1521/pedi.2011.25.3.397

Morey, L. C., Berghuis, H., Bender, D. S., Verheul, R., Krueger, R. F., \& Skodol, A. E. (2011). Toward a model for assessing level of personality functioning in DSM-5, part II: empirical articulation of a core dimension of personality pathology. Journal of Personality Assessment, 93(4), 347-353. doi: 10.1080/00223891.2011.577853

Raine, A., \& Benishay, D. (1995). The SPQ-B: a brief screening instrument for schizotypal personality disorder. Journal of Personality Disorders, 9, 346-355.

Richardson, G. E. (2002). The metatheory of resilience and resiliency. Journal of Clinical Psychology, 58(3), 307-321.

Rosler, M., Retz, W., Retz-Junginger, P., Thome, J., Supprian, T., Nissen, T., Stieglitz, R. D., Blocher, D., Hengesch, G., \& Trott, G. E. (2004). [Tools for the diagnosis of attention- deficit/hyperactivity disorder in adults. Self-rating behaviour questionnaire and diagnostic checklist]. Nervenarzt, 75(9), 888-895. doi: 10.1007/s00115-003-1622-2

Samuel, D. B., \& Widiger, T. A. (2008). A meta-analytic review of the relationships between the five-factor model and DSM-IV-TR personality disorders: a facet level analysis. Clinical Psychology Review, 28(8), 1326-1342. doi: 10.1016/j.cpr.2008.07.002

Schafer, J. L., \& Graham, J. W. (2002). Missing data: our view of the state of the art. Psychological Methods, 7(2), 147-177.

Schotte, C. K. W., \& de Doncker, D. (1994). ADP-IV Questionnaire. Antwerp, Belgium: University Hospital Antwerp.

Schotte, C. K. W., de Doncker, D. A., Dmitruk, D., Van Mulders, I., D'Haenen, H., \& Cosyns, P. (2004). The ADP-IV questionnaire: differential validity and concordance with the semi-structured interview. Journal of Personality Disorders, 18(4), 405-419. doi: 10.1521/pedi.18.4.405.40348

Schotte, C. K. W., de Doncker, D., Vankerckhoven, C., Vertommen, H., \& Cosyns, P. (1998). Self-report assessment of the DSM-IV personality disorders. Measurement of trait and distress characteristics: the ADP-IV. Psychological Medicine, 28(5), 1179-1188.

Schulz, P., \& Schlotz, W. (1999). [The Trier Inventory for the Assessment of Chronic Stress (TICS). Scale construction, statistical testing, and validation of the scale work overload]. Diagnostica, 45(1), 8-19.

Schulz, P., Schlotz, W., \& Becker, P. (2004). Trierer Inventar zum chronischen Stress (TICS). Göttingen: Hogrefe.

Schupp, J., \& Gerlitz, J.-Y. (2008). BFI-S: Big Five InventorySOEP. In A. Glöckner-Rist (Ed.), Zusammenstellung sozialwissenschaftlicher Items und Skalen (ZIS Version 12.00). Bonn: GESIS.

Skodol, A. E. (2012). Personality disorders in DSM-5. Annual Review of Clinical Psychology, 8, 317-344. doi: 10.1146/ annurev-clinpsy-032511-143131

Thomas, K. M., Yalch, M. M., Krueger, R. F., Wright, A. G., Markon, K. E., \& Hopwood, C. J. (2013). The convergent structure of DSM-5 personality trait facets and fivefactor model trait domains. Assessment, 20(3), 308-311.

Tyrer, P. (2005). The problem of severity in the classification of personality disorder. Joumal of Personality Disorders, 19(3), 309-314.

Tyrer, P., \& Johnson, T. (1996). Classification of personality disorder. The American Journal of Psychiatry, 153, 1593-1597.

Tyrer, P., Crawford, M., Mulder, R., Blashfield, R., Farnam, A., Fossati, A., Kim, Y.- R., Koldobsky, N., LecicTosevski, D., Ndetei, D., Swales, M., Clark, L. A., \& Reed, G. M. (2011). The rationale for the reclassification of personality disorder in the 11th revision of the International Classification of Diseases (ICD-11). Personality and Mental Health, 5(4), 246-259. 
Verheul, R., \& Herbrink, M. (2007). The efficacy of various modalities of psychotherapy for personality disorders: a systematic review of the evidence and clinical recommendations. International Review of Psychiatry, 19(1), 25-38. doi: 10.1080/09540260601095399

Verheul, R., Andrea, H., Berghout, C. C., Dolan, C., Busschbach, J. J., van der Kroft, P. J., Bateman, A. W., \& Fonagy, P. (2008). Severity Indices of Personality Problems (SIPP-118): development, factor structure, reliability, and validity. Psychological Assessment, 20(1), 23-34. doi: 10.1037/1040-3590.20.1.23

Watson, D., Clark, L. A., \& Tellegen, A. (1988). Development and validation of brief measures of positive and negative affect: the PANAS scales. Journal of Personality and Social Psychology, 54(6), 1063-1070.

Widiger, T. A. (2003). Personality disorder and axis I psychopathology: the problematic boundary of axis I and axis II. Journal of Personality Disorders, 17(2), 90-108.

Widiger, T. A., \& Mullins-Sweatt, S. N. (2009). Five-factor model of personality disorder: a proposal for DSM-V. Annual Review of Clinical Psychology, 5, 197-220. doi: 10.1146/annurev.clinpsy.032408.153542

van Wijk-Herbrink, M., Andrea, H., \& Verheul, R. (2011). Cognitive coping and defense styles in patients with personality disorders. Journal of Personality Disorders, 25(5), 634-644. doi: 10.1521/ pedi.2011.25.5.634

Wright, A. G., Thomas, K. M., Hopwood, C. J., Markon, K. E., Pincus, A. L., \& Krueger, R. F. (2012). The hierarchical structure of DSM-5 pathological personality traits. Journal of Abnormal Psychology, 121(4), 951-957. doi: 10.1037/a0027669

Yang, M., Coid, J., \& Tyrer, P. (2010). Personality pathology recorded by severity: national survey. British Journal of Psychiatry, 197(3), 193-199. doi: 10.1192/bjp. bp. 110.078956

Zuckerman, M. (1994). Behavioral expressions and biosocial bases of sensation seeking. Cambridge: Cambridge University Press.

Address correspondence to: Michael Pascal Hengartner, Department of Applied Psychology, Zurich University of Applied Sciences (ZHAW), PO Box, CH-8032 Zurich, Switzerland. Email: michaelpascal.hengartner@zhaw.ch 